\title{
Prevalência dos Fatores de Risco para Doença Cardiovascular em Funcionários do Centro de Pesquisas da Petrobras
}

\author{
Maria de Fátima Duarte Matos, Nelson Albuquerque Souza e Silva, Armando Jorge Marques Pimenta, \\ Antonio José Ledo Alves da Cunha \\ Rio de Janeiro, RJ
}

\begin{abstract}
Objetivo - Determinar a prevalência de fatores de risco para doença cardiovascular em funcionários do Centro de Pesquisas da Petrobras.
\end{abstract}

Métodos - Em estudo descritivo transversal, foram avaliados clínica e laboratorialmente, de março de 2000 e fevereiro de 2001, empregados do Centro de Pesquisas da Petrobras, tendo sido excluídos os que não compareceram à realização do exame médico periódico anual de 2000. Calculados o percentual da ocorrência dos fatores de risco e a média e o desvio padrão das variáveis bioquímicas, da pressão arterial e do índice de massa corpórea.

Resultados - De um total de 1.191 empregados, foram estudados 970, sendo 75,4\% homens e 24,6\% mulheres, com idade média de 42,2 anos. A prevalência de fatores de risco foi o sedentarismo $(67,3 \%)$, o colesterol $>200$ $m g / d l$ (56,6\%), o sobrepeso (42\%), a obesidade (17\%), a hipertensão arterial (18,2\%), o tabagismo $(12,4 \%)$ e o diabetes mellitus $(2,5 \%)$.

Conclusão - A elevada prevalência de fatores de risco para doença cardiovascular, em indivíduos jovens, alerta para a necessidade de adoção de programas de promoção de saúde e prevenção de doenças no ambiente de trabalho.

Palavras-chave: doença aterosclerótica, fatores de risco, ambiente de trabalho
Faculdade de Medicina da Universidade Federal do Rio de Janeiro

Correspondência: CENPES/SMS/SAUDE - Cidade Universitária - Quadra 7 -

Ilha do Fundão - Cep 21949-900 - Rio de Janeiro, RJ

E-mail:mfmatos@cenpes.petrobras.com.br

Recebido para publicação em 30/12/02

Aceito em 13/5/2003
Historicamente, o interesse da doença e suas complicações no ambiente de trabalho esteve focado no grau de exposição ocupacional, que leva aos perigos relacionados à saúde ${ }^{1}$

Nos últimos anos, esta ênfase tem mudado para doenças crônicas não-transmissíveis, e os locais de trabalho passaram a atrair interesse, como lugar potencial para estudos causais e de intervenções. Estes últimos têm como alvo a modificação de comportamentos precursores de doenças, não específicas da função ocupacional, como dieta, exercícios e tabagismo.

As doenças cardiovasculares têm papel indiscutível na morbidade e mortalidade do mundo ocidental, tanto nos países desenvolvidos como nos em desenvolvimento. A cardiopatia isquêmica e o acidente vascular cerebral são e serão, de acordo com as projeções para o ano 2020, as principais causas de morte, de anos de vida perdidos e de anos de vida perdidos com incapacitação ${ }^{2,3}$.

Em função da elevada prevalência das doenças cardiovasculares, dos poucos estudos nacionais, e da possibilidade de intervenção nos fatores de risco no ambiente de trabalho, optou-se por realizar esta pesquisa, com objetivo de analisar, nos empregados do Centro de Pesquisas da Petrobras, a prevalência dos fatores de risco para doença cardiovascular e, a partir daí, desenvolver ações de promoção da saúde.

\section{Métodos}

Trata-se de estudo transversal, realizado com empregados do Centro de Pesquisas da Petrobras, situado no Rio de Janeiro, de março de 2000 a fevereiro de 2001.

A Petrobras, com 38.908 empregados, atua de forma integrada e especializada nos segmentos relacionados à indústria de óleo, gás e energia e é o berço da tecnologia do petróleo brasileiro, cuja população é constituída de físicos, matemáticos, biólogos, geólogos, engenheiros, médicos, técnicos e administradores, reunindo 1.191 profissionais, entre mestres, doutores, gerentes com MBA (Master Business Administration), atuando em projetos de pesquisas ligados às áreas de exploração, produção e refino do petróleo nacional, além da busca de novas fontes alternativas de energia para o país. O Centro possui 137 laboratórios e 
um parque com 28 unidades-piloto (unidades para testes em refinarias).

Foram convocados 1.191 empregados para realizar o exame médico anual da empresa no período citado. Os empregados que compareceram, foram submetidos a exame clínico e laboratorial padronizados. O sangue foi coletado por técnico especializado de laboratório credenciado, e levado para análise no mesmo dia. O exame clínico foi realizado por médicos da empresa e as medidas antropométricas e da pressão arterial foram efetuadas por técnicos e auxiliares de enfermagem treinados, com equipamento para mensuração aferido regularmente.

Os valores do perfil lipídico foram analisados de acordo com o Segundo Programa Nacional de Educação do Colesterol dos EUA (NCEP) ${ }^{4}$, que classifica o colesterol total em normal $(<200 \mathrm{mg} / \mathrm{dl})$, limítrofe alto $(200-239 \mathrm{mg} / \mathrm{dl}) \mathrm{e}$ alto (>240 mg/dl); os triglicerídeos em normal ( $<200 \mathrm{mg} / \mathrm{dl})$, limítrofe alto (200-400 mg/dl), alto (400-1000 mg/dl) e muito alto $(>100 \mathrm{mg} / \mathrm{dl})$ e o HDL colesterol em baixo $(<35 \mathrm{mg} / \mathrm{dl})$, normal (35-59 mg/dl) e alto (>60 mg/dl).

A presença de familiares de $1^{\circ}$ grau com doença arterial coronariana manifesta e/ou cerebrovascular e/ou periférica, foi considerada como história familiar positiva.

O diagnóstico de diabetes mellitus (glicemia de jejum com valor $\geq$ a $126 \mathrm{mg} / \mathrm{dl}$ ) seguiu as normas do Expert Committee on Diagnosis and Classification of Diabetes Mellitus $^{5}$.

Os indivíduos que negavam prática regular de atividade física ou com frequiência $\leq 2$ vezes por semana, e duração mínima de 30min, foram tidos como sedentários.

Foram considerados fumantes indivíduos que fumavam, regularmente, em média, de três ou mais cigarros por dia, tragando a fumaça há $\geq 1$ ano.

De acordo com o índice de massa corpórea (IMC), os indivíduos foram classificados em: eutróficos (IMC $\geq 18 \mathrm{~m}^{2} \mathrm{e}$ $\leq 24,99 \mathrm{Kg} / \mathrm{m}^{2}$ ); sobrepeso (IMC $\geq 25 \mathrm{e} \leq 29,99 \mathrm{Kg} / \mathrm{m}^{2}$ ); obesidade $\left(\mathrm{IMC} \geq 30 \mathrm{Kg} / \mathrm{m}^{2}\right)^{6}$.

Em relação à pressão arterial, os valores de referência adotados obedeceram às recomendações do Joint National Committee VI(JNC VI) ${ }^{7}$, que define como hipertensão arterial níveis $\geq 90 \mathrm{mmHg}$ de pressão diastólica e $\geq 140 \mathrm{mmHg}$ de pressão sistólica. As pessoas que estavam com pressão arterial abaixo desses níveis, mas referiam uso de drogas anti-hipertensivas, também foram consideradas hipertensas.

Após avaliação clínica e laboratorial foi realizada análise estatística descritiva, calculado o valor mínimo, primeiro quartil, mediana, terceiro quartil, máximo, média e desvio padrão para as variáveis bioquímicas, para a pressão arterial e para o índice de massa corpórea e calculado o valor percentual dos fatores de risco.

\section{Resultados}

Dos 1.191 empregados convocados, 970 compareceram e foram avaliados, sendo $75,4 \%$ homens e $24,6 \%$ mulheres, com idade média de 42,2 anos.

Dados referentes ao perfil laboratorial encontram-se na tabela 1 e referentes à pressão arterial e índice de massa corpórea na tabela II. Na figura 1 está a prevalência dos principais fatores de risco.

\begin{tabular}{|cccccc|}
\hline \multicolumn{6}{|c|}{ Tabela I - Níveis das variáveis bioquímicas dos 970 empregados do } \\
CENPES, Rio de Janeiro, 2000 \\
\hline $\begin{array}{c}\text { Estatísticas } \\
\text { Descritivas }\end{array}$ & $\begin{array}{c}\text { Glicemia } \\
(\mathrm{mg} / \mathrm{dl})\end{array}$ & $\begin{array}{c}\text { Colesterol } \\
(\mathrm{mg} / \mathrm{dl})\end{array}$ & $\begin{array}{c}\text { HDL } \\
(\mathrm{mg} / \mathrm{dl})\end{array}$ & $\begin{array}{c}\text { LDL }^{*} \\
(\mathrm{mg} / \mathrm{dl})\end{array}$ & $\begin{array}{c}\text { Triglicerídeos } \\
(\mathrm{mg} / \mathrm{dl})\end{array}$ \\
\hline Média & 91,9 & 208,4 & 48,1 & 132,3 & 143,3 \\
Desvio-padrão & 16,1 & 41,3 & 11,5 & 36,5 & 127,1 \\
Mínimo & 65 & 107 & 22 & 20,8 & 32 \\
$1^{\circ}$ quartil & 85 & 179 & 40 & 107,2 & 76 \\
Mediana & 90 & 205 & 46 & 130,6 & 109 \\
$3^{\circ}$ quartil & 95 & 231 & 55 & 151,8 & 165 \\
Máximo & 299 & 376 & 110 & 297,6 & 1,725 \\
\hline \multirow{2}{*}{ n=967 } & \multicolumn{5}{c}{} \\
\hline
\end{tabular}

\begin{tabular}{|cccc|}
\hline \multicolumn{4}{|c|}{ Tabela II - PA Sistólica, PA Diastólica e IMC dos 970 empregados do } \\
CENPES, Rio de Janeiro, 2000 \\
\hline Estatísticas & $\begin{array}{c}\text { PA Sistólica } \\
(\mathrm{mmHg})\end{array}$ & $\begin{array}{c}\text { Pa Diastólica } \\
(\mathrm{mmHg})\end{array}$ & $\begin{array}{c}\mathrm{IMC} \\
\left(\mathrm{Kg} / \mathrm{m}^{2}\right)\end{array}$ \\
Descritivas & 116,4 & 75,7 & 26,5 \\
\hline Média & 15,1 & 10,5 & 4,5 \\
Desvio-padrão & 80 & 50 & 16,9 \\
Mínimo & 110 & 70 & 23,6 \\
$1^{\circ}$ quartil & 120 & 80 & 25,8 \\
Mediana & 120 & 80 & 28,6 \\
$3^{\circ}$ quartil & 230 & 140 & 51,6 \\
Máximo & & \\
\hline
\end{tabular}

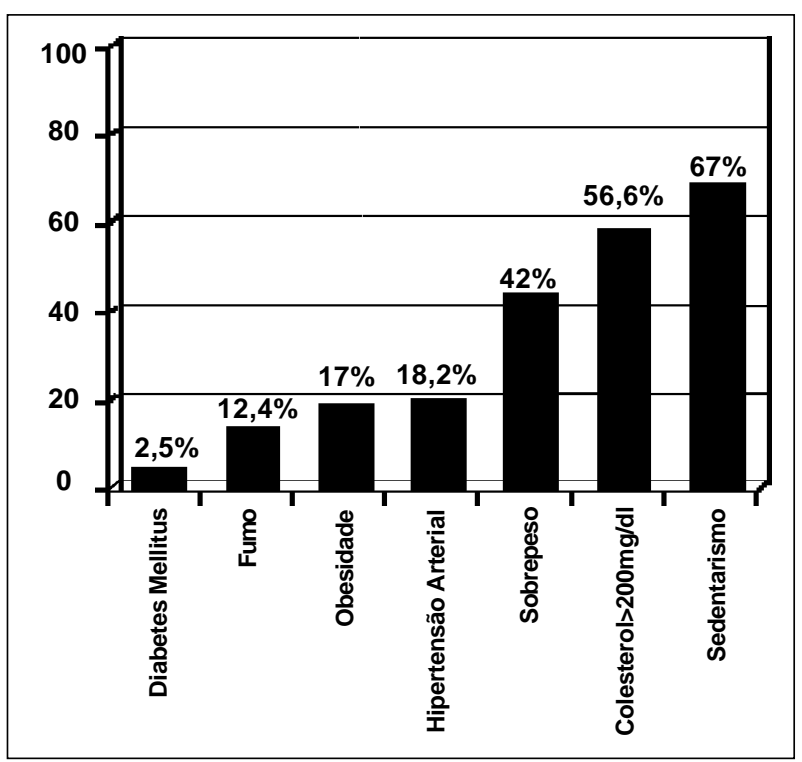

Fig. 1 - Prevalência dos principais fatores de risco nos 970 empregados do CENPES, Rio de Janeiro, 2000.

O sedentarismo, presente em $67,3 \%$ da amostra, destacou-se como fator de risco mais prevalente. A obesidade foi observada em $17 \%$ da população, sendo que $42 \%$ encontravam-se com sobrepeso. A hipertensão arterial esteve presente em $18,2 \%$ dos empregados, sendo que apenas $16 \%$ dos hipertensos encontravam-se controlados. O tabagismo foi observado em $12,4 \%$ da amostra e o diabetes mellitus em $2,5 \%$.

Entre os antecedentes familiares, observou-se que para doença arterial coronariana, 25,5\% possuíam história 
positiva; para hipertensão arterial sistêmica e acidente vascular cererbral, $45,6 \%$ e $12,5 \%$, respectivamente.

Níveis de colesterol total acima dos valores normais (limítrofe alto e alto) foram observados em $56,6 \%$ dos indivíduos, sendo 19,3\% superiores a 240mg/dl. Níveis diminuídos de HDL colesterol foram encontrados em $8,7 \%$ da população e níveis altos de colesterol HDL em 15,4\%. Níveis de triglicerídeos acima de $200 \mathrm{mg} / \mathrm{dl} \mathrm{em} 17 \%$.

\section{Discussão}

Durante muito tempo, persistiu a idéia de que as doenças cardiovasculares seriam determinadas geneticamente, $\mathrm{e}$ que pouco poderia ser feito para sua prevenção. Porém, o reconhecimento dos fatores de risco modificou essa visão.

Os resultados iniciais do primeiro projeto de intervenção nos hábitos de vida de um grupo populacional, realizado na Karélia do Norte, província finlandesa com altíssimas taxas de incidência e de mortalidade por doença arterial coronariana, mostraram a possibilidade de reduções importantes nesses índices, através de ações abrangentes relacionadas ao estilo de vida ${ }^{8}$.

Apesar do grupo estudado ser bastante diferenciado, apresentou índices bem semelhantes a outras populações, independente do nível sócio-econômico-cultural.

No perfil lipídico, observou-se que $55,6 \%$ apresentavam colesterol $>200 \mathrm{mg} / \mathrm{dl}$, dos quais $19,1 \%$ colesterol $\geq 240 \mathrm{mg} / \mathrm{dl}^{9}$. Prevalência até mais elevada do que a da população norte-americana, onde se estima que 51,8\% apresentam colesterol $>200 \mathrm{mg} / \mathrm{dl} \mathrm{e} 20 \%>240 \mathrm{mg} / \mathrm{dl}^{4}$. A média de colesterol total foi de $207,6 \mathrm{mg} / \mathrm{dl}$, também superior à observada em alguns estudos nacionais. Ducan e cols. ${ }^{10}$ encontraram um colesterol médio de $202,4 \mathrm{mg} / \mathrm{dl}$ e o estudo realizado em Cotia, município da grande São Paulo ${ }^{11}$, apresentou uma média de $184,9 \mathrm{mg} / \mathrm{dl}$. Souza ${ }^{12}$, em população de motoristas de ônibus, com idade média semelhante à população do Centro de Pesquisa da Petrobras, encontrou média de $211,6 \mathrm{mg} / \mathrm{dl}$.

A presença de níveis elevados de índice de massa corpórea foi outro aspecto preocupante nesta população. Encontraram-se $42 \%$ dos indivíduos com sobrepeso e $16 \%$ obesos, ou seja, 58\% com excesso de peso. A média de índice de massa corpórea foi de $26,3 \mathrm{Kg} / \mathrm{m}^{2}$ e no estudo de Sou$\mathrm{za}^{12} 25,8 \mathrm{Kg} / \mathrm{m}^{2} \mathrm{e} 59,5 \%$ de indivíduos com sobrepeso. Dados do Consenso Latino-Americano de Obesidade ${ }^{13}$ mostraram que na Argentina 32,5\% da população estava com sobrepeso e $27 \%$ com obesidade; no Uruguai, $42 \%$ com excesso de peso, e no Brasil, 53\%. NosEUA, em $2000^{14}$, observou-se que $19,8 \%$ da população encontravam-se na faixa de obesidade.

Com relação ao tabagismo, observou-se que $69,4 \%$ da população nunca havia fumado, $18,2 \%$ eram ex-fumantes e apenas $12,4 \%$ tabagistas, valores muito inferiores aos observados na literatura. Souza ${ }^{12}$ encontrou uma prevalência de 32,7\%. Na população da Ilha de Governador observou-se uma prevalência de $33 \%{ }^{15}$. Moreira e cols. ${ }^{16} \mathrm{e}$ Pohlmann e cols. ${ }^{17}$ observaram prevalência de $41,5 \%$ e $45,7 \%$ respecti- vamente. No mundo, estima-se que $1 / 3$ da população adulta seja fumante. A baixa prevalência deste fator de risco na população em estudo deve-se, provavelmente, ao trabalho intenso de convencimento para o abandono do hábito, que vem sendo desenvolvido pela empresa há aproximadamente 10 anos.

O sedentarismo foi mais um fator de risco prevalente. Encontraram-se 67,4\% dos indivíduos sedentários e apenas $32,6 \%$ ativos. Estudo envolvendo 37 estados americanos demonstrou que $58 \%$ dessa população é sedentária ${ }^{18}$. No Estado de São Paulo ${ }^{19}$, o sedentarismo atinge 69\% da população e no estudo de Souza, $86 \%{ }^{12}$.

A prevalência de diabetes mellitus foi de 2,5\% ( $n=24)$, inferior à observada na literatura, considerando-se a idade média da população. Souza ${ }^{12}$, em uma população com idade média de 41,3, encontrou prevalência de 7,32\%.

A prevalência de hipertensão arterial na população foi de $18,2 \%$. Esta prevalência varia amplamente no mundo, com valores próximos de $4 \%$ na China, e em torno de $20 \%$ nos EUA ${ }^{20}$. Recentemente, foi realizado um levantamento epidemiológico e clínico entre os moradores da XX Região Administrativa do Município do Rio de Janeiro (Ilha do Governador) e encontrados $38 \%$, bem acima do que é descrito em outras populações ${ }^{15}$, sendo o de Souza ${ }^{12} 20,7 \%$.

Lessa $^{21}$ apresentou uma revisão de 51 estudos brasileiros (incluindo crianças e adolescentes) sobre a prevalência da hipertensão arterial, realizada entre 1970 e meados de 1993. As diferenças entre as prevalências mencionadas no Brasil são muito amplas, variando entre 7,2\% e 40,3\% no Nordeste; $5,04 \%$ a $37,9 \%$ no Sudeste; $1,28 \%$ a $27,1 \%$ no Sul e $6,3 \%$ a $16,75 \%$ no Centro-Oeste, devidas, em parte, à diversidade de características metodológicas importantes entre os estudos.

A falta de pesquisas sobre fatores de risco na população brasileira resulta em grande desvantagem em relação aos países desenvolvidos. No caso da população economicamente ativa, a falta de dados é dramática, mesmo em setores de grande importância econômica.

Com relação às limitações do estudo, ressaltamos que foram observados os cuidados necessários para redução de erros sistemáticos, através do treinamento da equipe técnica envolvida, bem como aferição dos equipamentos utilizados.A respeito dos 221 empregados excluídos, que por motivos diversos não puderam comparecer ao exame, supõe-se que não difiram substancialmente da amostra total, dado que a idade média e a distribuição por sexo foram semelhantes à observada entre os 970 empregados estudados.

Apesar dos resultados obtidos serem muito semelhantes aos observados na literatura em populações distintas, as características peculiares do grupo pesquisado não permitem a generalização das observações para a população geral.

Pelos dados encontrados pode-se inferir que os empregados do Centro de Pesquisa da Petrobras apresentam risco moderado para o desenvolvimento de doenças cardiovasculares, apesar da baixa faixa etária, do nível de escolaridade elevado e dos recursos disponíveis na empresa.

Levando-se em conta que é freqüente a existência de três, quatro ou mais fatores de risco em um mesmo paciente, 
altamente relacionados entre si, nãoé difícil compreender a necessidade de intervenções múltiplas. Como o ser humano passa cerca de $65 \%$ de seu tempo de vida no ambiente de trabalho, torna-se o local ideal para que ele aprenda o que não aprendeu na escola ou com a família, já que só a partir da educação é possível efetivamente prevenir doenças. Assim, o desenvolvimento de programas de saúde de caráter preventivo, com enfoque na mudança do estilo de vida, pode ser um meio eficaz para sensibilizar o indivíduo quanto à mudança de hábitos de vida nocivos à saúde.

\section{Referências}

1. Ramazzini B. De Morbis Artificum Diatriba. In: Estrela R. As Doenças dos Trabalhadores. São Paulo: Ministério do Trabalho, Fundacentro, 1985:9-179.

2. Murray CJL.The Global Burden of Disease. WHO, HSPH, W. BANK -1996

3. Levy D, Wilson PWF. Atherosclerotic cardiovascular disease - an epidemiologic perspective. In: Topol EJ, editores. Textbook of Cardiovascular Medicine. $2^{\mathrm{a}}$ ed. Philadelphia: Lippincott-Raver;1998:13-29.

4. National Cholesterol Education Program. Second report of the Expert Panel on Detection, Evaluation and Treatment of High Blood Cholesterol in Adults (Adult Treatment Panel II).Circulation 1994; 89: 1329-445.

5. The Expert Commitee on the Dignosis and Classifiction of Diabetes Mellitus. Report of the expert commitee on the dignosis and classifiction of diabetes mellitus. Diabetes Care 1997;20:1183-97.

6. World Health Organization(WHO). Physical Status: the use and interpretation of anthropometry. Geneve: WHO, 1995. (Technical Report Series, 854).

7. Joint National Commitee on Prevention, Detection, Evaluation, and Treatment High Blood Pressure. The sixth report of the Joint National Commitee on Prevention, Detection, Evaluation, and Treatment High Blood Pressure. Arch Intern Med 1997;157:2413-46.

8. Puska P, Tuomileto J, Salone J, et al. Community control of cardiovascular diseases. Evalution of a comprehensive community programme for control of cardiovascular diseases in North Karelia, Finland.1972-1977, WHO:Copenhagen, 1981.

9. Forti N, Fukushima J, Gianini SD. Perfil lipídico de indivíduos submetidos à cinecoronariografia em diferentes regiões do Brasil. Arq Bras Cardiol 1997;68:333-42.

10. Duncan BB, Berger C, Silva ML, et al. Níveis séricos de colesterol em amostra representativa da população adulta de Porto Alegre. Arq Bras Cardiol 1988; 51:385-90.

11. Martins IS. Doenças cardiovasculares ateroscleróticas, dislipidemias, hiperten- são, obesidade e diabetes mellitus em população de área metropolitana da região sudeste do Brasil: II-dislipidemias. Rev Saúde Pública 1996; 30: 75-84.

12. Souza NRM. Fatores de risco cardiovascular em motoristas de ônibus [Dissertação de de Mestrado]. Niterói: Universidade Federal Fluminense, 1999:271

13. Coutinho W. Consenso Latino-Americano em obesidade. Disponível na INTERNET via www.abeso.org.br/consenso.doc. Arquivo consultado em 2001.

14. Pi-Sunyer FX. NHLBI- Obesity education initiative expert panel on the identification, evaluation, and treatment of overweight and obesity in adults. Disponível na INTERNET via www.cdc.gov. Arquivo consultado em 2001.

15. Klein CH, Silva NAS, Nogueira AR, Campos CHS, Bloch KV. Relatório de Pesquisa de Hipertensão Arterial na Ilha do Governador. HUCFF-UFRJ/Ministério de Saúde, 1992.

16. Moreira LB, Fuchs FD, Moraes RM et al. Prevalência de tabagismo e fatores associados em área metropolitanada região sul do Brasil. Rev Saúde Pública 1995; 29:46-51.

17. Pohlmann PR. Tabagismo em Porto Alegre: prevalência eo papel dos profissionais da saúde na prevenção. Rev Ass Med Brasil 1991; 37: 8-14

18. National Heart Institute. Physical activity and cardiovascular health. JAMA 1996; 276: 241-6.

19. Rego RA, BerardoFAN, Rodrigues SSR et al. Fatores de risco para doenças crônicas não-transmissíveis: inquérito domiciliar no município de São Paulo, SP(Brasil). Rev Saúde Pública 1990; 24: 277-85.

20. Porto CC. Hipertensão Arterial. In: Porto CC editores. Doenças do coração- prevenção e tratamento.Rio de Janeiro: editora Guanabara Koogan, 1998:453-60.

21. Lessa 1. Estudos Brasileiros Sobre a Epidemiologia da Hipertensão Arterial: Análise Crítica dos Estudos de Prevalência. Inf. Epidemiol., SUS, Cenepi, Ministério da Saúde 1993; 3: 59-75. 